\title{
EL MUSEO DEL CARIBE, UN LABORATORIO DE DISEÑO PARA LA PRIMERA INFANCIA.
}

\section{MUSEO DEL CARIBE, A DESIGN LABORATORY FOR EARLY CHILDHOOD}

\author{
FECHA DE RECIBO: OCTUBRE 31, 2012 \\ FECHA DE ACEPTACIÓN: DICIEMBRE 20, 2012 \\ DIANA CASALINS \\ Maestra en Artes Plásticas, Jefe de Departamento de Educación, \\ dcasalins@culturacaribe.org. Grupo “Cultura Caribe”.dcasalins@culturacaribe.org. \\ TATIANA MIRANDA \\ Licenciada en Pedagogía Infantil, Profesional de Apoyo en Proyectos Educativos. \\ tmiranda@culturacaribe.org. Grupo "Cultura Caribe”.tmiranda@culturacaribe.org
}

\begin{abstract}
"El Museo del Caribe, un laboratorio de diseño para la primera infancia" es una investigación de la Corporación Parque Cultural del Caribe, desarrollada durante 2012 por el Grupo de investigación "Cultura Caribe". Este proyecto fue desarrollado con el apoyo de diversas entidades y personas: Ministerio de Cultura de la República de Colombia; Mg. Reinaldo Niebles De la Torre; y la Universidad Autónoma del Caribe a través de los investigadores del Grupo Ellipsis del Programa de Diseño Gráfico de la Facultad de Arquitectura Arte y Diseño, Profesores Ketty Miranda, Ernesto Monroy y Leonardo Infante; y las madres, padres y maestros asistentes a las sesiones de laboratorio.
\end{abstract}

\section{RESUMEN:}

Los primeros años de vida suponen un gran impacto en la vida del ser humano. Por ello, vemos un significativo enfoque hacia programas y actividades dirigidos al trabajo con la primera infancia. Para el Museo del Caribe, el diseño de programas para niños de cero a seis años reveló, por una parte, las tendencias naturales de las actividades ofrecidas por los museos para este público, y por otro que las expectativas de padres y maestros no siempre se alinean con las primeras. En esta dirección, se construyó un portafolio de actividades educativas, realizando un proceso concertado con docentes, madres, artistas, psicólogos y diseñadores gráficos e industriales, laboratorios de investigación, diseño y prototipo. Como resultado, se consolidó un método de trabajo que articula eficazmente la participación de los actores inmersos en el proceso, con el fin de obtener resultados más pertinentes y auténticos. Este Artículo invita a la reflexión sobre el nuevo significado educativo de los museos, cada vez más activos en la formación en la primera infancia.

\section{PALABRAS CLAVE:}

museos, educación formal, educación informal, conocimiento científico, primera infancia, interdisciplinariedad, diseño.

\section{ABSTRACT:}

The inclinations on learning for human beings are crucial in early years, this is the main reason why there is a substantial focus on programs and activities designed for teaching in the early childhood. The "MUSEO DEL CARIBE" from Barranquilla, in their studies within children from zero to six years old, revealed a tendency where the activities offered by museums and the expectations parents and teachers have from their visit, are not equal. Looking forward within the studies, a program was built, where parents, artists, psychologists, graphic and industrial designers will come with an ideal method to articulate the participation of the actors involved in the process, and a search of more authentic and relevant outcomes. This article invite us to reflect about new educational purpose fullness of museums, increasingly active for children.

\section{KEYWORDS:}

museums, formal education, informal education, scientific knowledge, early childhood, interdisciplinarity, design. 


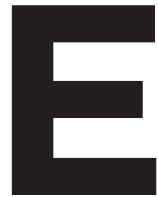

xiste un consenso entre comunicadores, educadores y especialistas en museología: los museos, entendidos, como espacios prestadores de servicios culturales dirigidos a todo tipo de público, dejando atrás aquella oferta destinada solamente a académicos, profesionales, investigadores y profesores. Hoy en día, los museos están enfocando su oferta al mercado del ocio cultural, en general a todas aquellas personas que busquen el disfrute y el aprovechamiento de su tiempo libre (Ojeda, 2008)

Aunque en sus orígenes los museos no habían sido creados pensando en los escolares como grupo mayoritario, se han hecho frecuentes las visitas de grupos escolares constituyéndose alrededor del $50 \%$ del público visitante. Las visitas escolares de niños entre los cinco y 16 años de edad, son cada vez más frecuentes con el objetivo de complementar o profundizar en el estudio de determinados aspectos de su aprendizaje.

Ante esta realidad, los museos han visto la necesidad de renovarse y de remover sus estructuras para ir más allá de la custodia y la colección, para lograr que sus exposiciones adquirieran nuevos sentidos y significados; por ello, están incursionando en ofrecer a este tipo de público, dispositivos interactivos y actividades educativas que vayan de acuerdo con sus expectativas y necesidades, logrando que el visitante escolar deje de ser un observador para convertirse en un participante.

Este artículo pretende mostrar cómo el Museo del Caribe de Barranquilla se prepara para ofrecer al público de la primera infancia espacios educativos que les permita aprender y vivir experiencias que fomenten el conocimiento, apreciación, disfrute, apropiación, valoración y conservación del patrimonio natural y cultural del Caribe Colombiano.

\section{¿POR QUÉ DISEÑAR PARA PRIMERA INFANCIA EN UN MUSEO?}

“Cada sociedad, cada cultura y cada época definen explícita o implícitamente qué es la infancia, cuáles son sus características $y$, en consecuencia, qué períodos de la vida incluye. Por lo tanto, es importante entender que nuestra imagen de los niños es una construcción social creada por el hombre, que surge de nuestras expectativas, y que los niños no son niños «por naturaleza», sino que, de hecho, desde la perspectiva histórica, la consideración de los niños como categoría social diferente, es una creación muy reciente" (Ancheta, 2008, p.1-2).

En el caso de Colombia, las ideas en torno a la primera infancia han variado de acuerdo a diferentes aspectos sociales, económicos, políticos, pedagógicos y culturales propios de cada época histórica. Para ilustrar, a principios del siglo XX la primera infancia se debatía entre una concepción de los niños como pequeños adultos, y la idealización de estos como cándidas tabulas rasas que debían ser moldeadas por sus tutores y maestros.
No obstante, el movimiento y complejidad del mundo actual, permitió que la infancia se concibiese como una categoría que circunscribe un mundo de experiencias y expectativas distintas a las del mundo adulto. Es así como a partir de la Convención Internacional de los Derechos del Niño, aprobada por la Asamblea General de las Naciones Unidas el 20 de noviembre de 1989, se le define como un sujeto de derecho. En tal sentido, se les reconoce a los infantes su estatus de persona y de ciudadano. Pensar en los niños como ciudadanos es reconocer igualmente los derechos y obligaciones de todos los actores sociales.

En el contexto colombiano actual, con la publicación de la Política Pública de Primera Infancia, en el año 2006, se señala que el niño es un ser único, con una especificidad personal activa, biológica, psíquica, social y cultural en expansión; y con base en el Código de la Infancia y la Adolescencia, la primera infancia es el periodo que comprende la franja poblacional que va desde los cero a los seis años de edad, y es la etapa del ciclo vital en la que se establecen las bases para el desarrollo cognitivo, emocional y social del ser humano.

\section{« Hoy en día, los museos están enfocando su oferta al mercado del ocio cultural, en general a todas aquellas personas que busquen el disfrute y el aprovechamiento de su tiempo libre.»}

En otras palabras, los niños nacen con sofisticadas capacidades y en poco tiempo construyen mejores herramientas para pensar, procesar y transformar la información que reciben de su entorno y aprenden rápidamente de las relaciones que se tejen en el contexto familiar y en todos los ambientes sociales donde conviven (MEN, 2009, p.12). La primera infancia es una etapa muy importante en la vida de todo ser humano, es un periodo en el que se llevan a cabo procesos de reorganización y de transformación permanente de competencias y que por tal motivo demanda de experiencias significativas que propicien el conocimiento del mundo real, de los otros y de sí mismos (MEN, 2009).

Si bien en la actualidad se ha ampliado el rol de los museos a la acción consciente de educar, transmitir y comunicar, algunas de sus tareas históricas prevalecen. El museo sigue siendo un centro de comunicación en cuanto sirve de canal para que las diferentes sociedades transmitan y comuniquen sus rasgos culturales a las generaciones venideras. De esta manera, preserva los diversos contenidos culturales, en un espiral cíclico en el que no solo se transmiten conocimientos a los 
jóvenes, sino que éstos se enriquecen al reproducirlo y construyen nuevos saberes. Como en un principio, y aunque a veces pretendan no hacerlo, los museos constantemente construyen memorias y versiones de las sociedades. Aparentan transmitir un pasado, pero lo que hacen es construir representaciones de ese pasado, y esas representaciones siempre res-ponden al presente en el que se construyen (Jaramillo, 2008)

En este proceso dinámico el museo se ha convertido en uno de los principales agentes educativos de las sociedades, situación que los pone en la necesidad de diferenciarse e identificarse ante las otras instituciones académicas. El camino lógico es distanciarse de las instituciones regulares y, por ende, de los métodos tradicionales de enseñanza. Puede apoyar la acción educativa de otras instituciones, pero no convertirse en recurso didáctico de las mismas. (Jaramillo, 2008)

Como lo dice Janette Griffin, "es vital para los museos que los jóvenes visitantes tengan experiencias memorables, porque esto influencia sus contratos futuros con las oportunidades culturales. Las buenas y tempranas experiencias determinaran sus propias actitudes en la participación que después tengan junto con las de sus familias y amigos. $Y$ de esto trata nuestra versión del efecto mariposa. Traducida, nuestra metáfora significa: pequeños aleteos, es decir, experiencias memorables en museos, que tal vez puedan tener gran impacto en la vida futura de quien la vivencia". (Citado por Alderoqui, 2008)

\section{¿QUÉ ESTAN HACIENDO LOS MUSEOS EN EL MUNDO PARA LA PRIMERA INFANCIA?}

Los museos y centros de ciencias juegan un papel relevante en la construcción de imaginarios y generación de espacios de aprendizaje significativo, sin embargo, apenas en los últimos 20 años se han empezado a generar iniciativas de diseño museal para la primera infancia; un público que resulta tan particular como diverso, y en el cual las instituciones museales y centros de ciencia no habían centrado su atención.

En palabras de Dufresne-Tassé (2008) “los educadores museales comenzaron a interesarse en los niños de edad preescolar en la medida en que los servicios educativos diversificaron su oferta para los más grandes. En esta dirección, los educadores desarrollaron programas, exposiciones e incluso museos caracterizados por una máxima utilización de la "interactividad", dirigidos a los pequeños, que en algunas ocasiones buscaban entretener mediante exhibits, pero que sobre todo, pretendían complementar el desarrollo psicomotor del niño o iniciarlo en el mundo del arte y de la ciencia" (p.56).

Hace 50 años, los museos ofrecían al público escolar visitas guiadas adaptadas al nivel escolar de los estudiantes. Como primer tipo de colaboración entre el museo y la escuela se desarrollaron e implementaron los maletines pedagógicos. Luego basados en el constructivismo, los museos empezaron a implementar las visitas taller o visitas rally. (Dufresne-Tassé, 2008).

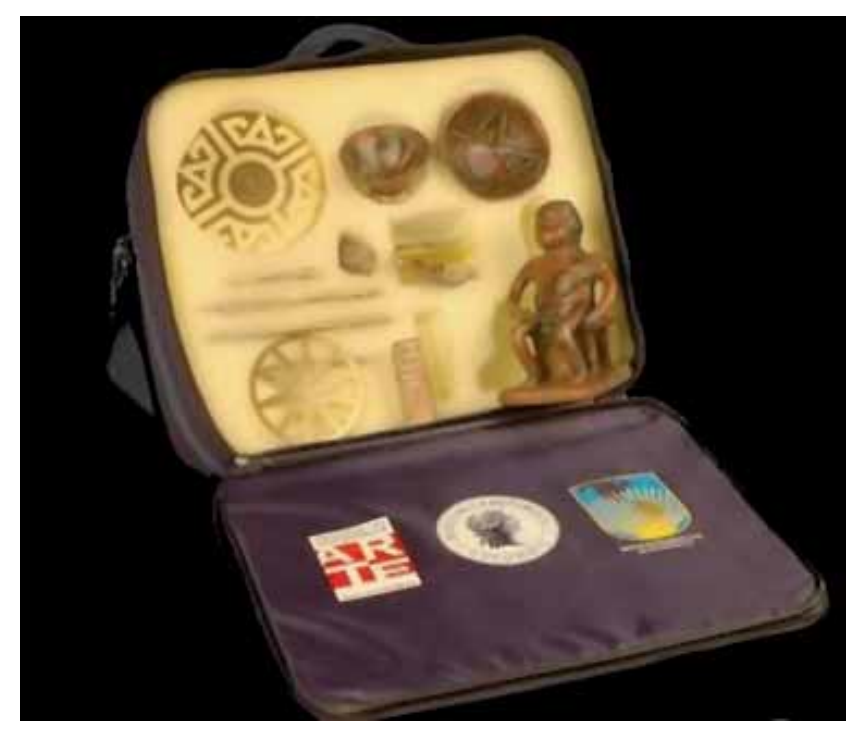

Maleta didáctica del Museo del Oro

FUENTE: Archivo Fotográfico, Museo del Oro. Bogotá, DC, 2010

Después, en los años 80, educadores y equipos educativos de museos desarrollaron investigaciones enfocadas en evaluar los tipos de visitas que se realizaban dentro de los museos. Estos estudios arrojaron que las visitas más significativas eran aquellas "bien hechas" y que se adaptaban a los escolares a quienes estaban dirigidas. A su vez, se logró verificar que las visitas eran más productivas cuando los profesores realizaban actividades complementarias antes y después de visitar el museo. Por ello, con el fin de seguir afianzando la relación museo-escuela, los educadores museales desarrollaron documentos de preparación y de seguimiento de la visita (Dufresne-Tassé, 2008).

\section{« Los museos y centros de ciencias juegan un papel relevante en la construcción de imaginarios y generación de espacios de aprendizaje significativo, sin embargo, ape- nas en los últimos 20 años se han empe- zado a generar iniciativas de diseño museal para la primera infancia.»}

“La experiencia reciente en diversos países muestra cómo los mismos usuarios de los museos contemporáneos (niños, jóvenes y adultos por igual) demandan una oferta versátil que les permita, mediante la exploración del museo, tener a la vez una oportunidad inspiradora de conocimiento, y una posibilidad de diversión y vivencia del tiempo libre de alto nivel o de un orden distinto y una experiencia en la que se pueda desa- 
tar su creatividad a partir de los diversos estímulos" (Orozco, 2005, p.39). Por consiguiente, con el objetivo de garantizar el acceso a espacios educativos, en las que se les permita a los niños explorar y enriquecer sus conocimientos, los museos han emprendido un camino inscribiendo sus prácticas cada vez más en el marco del aprendizaje informal, dentro del cual la experimentación, el juego, la interacción, la posibilidad de conocer directamente objetos y espacios patrimoniales o de ciencia, constituyen el centro mismo de la experiencia museal.

Es así como los museos convierten sus salas en espacios de exploración y su colección en herramientas de Object Based Learning, una metodología que facilita el acercamiento al conocimiento sobre temas complejos involucrando varios sentidos en una experiencia de aprendizaje activo (Biggs, 2003; Prince, 2004), o hacen uso de sus educadores con técnicas de interpretación del Patrimonio (Heritage Interpretation), que tienen por objetivo conectar los discursos y experiencias desarrolladas dentro de los museos, con las experiencias cotidianas de sus visitantes.

\section{« Los museos mantienen, como idea, que cada exposición debe estar en consonancia con el perfil del usuario común, no de un usuario ideal 0 abstracto, si bien al inicio el conocimiento de sus niveles y estilos cog- noscitivos sea mínimo o preliminar.»}

En este sentido, los museos han concentrado esfuerzos en crear espacios significativos de aprendizaje para la primera infancia, que de acuerdo a lo planteado por el MEN (2009) no deben entenderse como un lugar que remite a lo físico, sino a aquellas experiencias que están en capacidad de ser: 1) una situación estructurada, 2) un contexto de interacción, 3) una situación de resolución de problemas, y 4) una situación que exija el uso de competencias variadas. En la medida en que estos "espacios" alcancen a integrar estas cuatro condiciones, se desarrolla un aprendizaje significativo para los participantes.

Sin embargo, las tendencias de diseño que se han seguido y la oferta específica para los niños más pequeños, muchas veces reflejan la falta de escrutinio y el uso de elementos culturales contextuales que potencien las experiencias. Se entiende que un espacio educativo es significativo si se trata de cualquier situación, actividad, tarea, problema o práctica cultural que les brinda la oportunidad de aprender, movilizar sus competencias y que realmente les exija "pensar" (MEN, 2009).

En una primera revisión de la oferta actual para primera infancia, en diferentes museos alrededor del mundo, realizada por el equipo de educación del Museo del Caribe utilizando la información disponible en las páginas web de los museos consultados, se encontraron rápidamente algunas tendencias.

A continuación se mencionan las más frecuentes:

1. No siempre se piensa en primera infancia al pensar en niños. Algunos museos poseen una oferta de actividades para niños que empieza en edad escolar (cinco ó seis años en adelante). Una actividad típica, que aparece etiquetada como disponible para niños son recorridos temáticos ("La caza del león" - recorriendo la iconografía alrededor de este felino a través de las obras del museo), que requieren la compañía de adultos, procesamiento de lenguaje y otras características que dificultan la exploración autónoma o no explotan los intereses de los niños más pequeños por tocar, oler, morder... (Ej: recorridos temáticos para niños. Museu du Louvre)

2. Cuentos en el museo: Existe una tendencia en los museos que asocia actividades infantiles con sesiones de lectura de cuentos, muchas de las actividades desarrolladas en los museos se enfocan en desarrollar experiencias alrededor de una lectura en la que el mediador motiva la participación de los niños. La ventaja de esta modalidad de actividades consiste en que muchos museos permiten la participación de los más pequeños (desde 18 meses de edad), con la compañía de sus padres (Ej: MET, Museum. Family Programs)

3. Tocar, oler, oír: En una creciente conciencia en torno a las necesidades de los niños, empiezan a parecer actividades sofisticadas concentradas en los sentidos: ver, tocar, oler, probar... La ventaja de estas actividades sensoriales consiste en la variedad de posibilidades que se presentan a los niños, la desventaja consiste en la necesidad de crear un espacio o exhibiciones especiales que permitan la exploración libre de los niños a través de los diversos objetos y materiales propuestos (Ej. MOMA, Kids and families lab).

4. Recorrido para niños: En muchos museos, es frecuente ofrecer visitas acompañadas para niños, estas consisten básicamente en una interpretación que busca acercar a los grupos de los más pequeños a hacerse preguntas en torno a las obras de las colecciones. Como ventaja ofrecen la moderación del nivel de complejidad del lenguaje utilizado y el hecho de permitir a pequeños grupos conocer las colecciones, como desventaja, estas visitas no se encuentran disponibles para los más pequeños por las dificultades que plantea (Ej: Thyssen Bornemisza, Visitas dinamizadas)

5. Juegos de roles: Una de las opciones que se presenta con relativa frecuencia es el juego de roles, "jugar a ser..." En estas actividades, se cuenta con un mediador que ayuda y orienta a los niños participantes, a quienes se les da una misión para desarrollar. Como ventaja, al ser una experiencia de inmersión a través del juego, logra que los niños disfruten la experiencia y adquieran conocimientos sobre un tema en específico. Como desventaja, muchos de estos 
juegos requieren como condición la participación de niños mayores de cinco años, e incluso, niños que sepan leer o escribir, lo cual remite nuevamente a la idea de "no siempre se piensa en primera infancia al pensar en niños" ( Ej: Natural History Museum UK. Wildlife garden explorer)

6. Visita autoregulada: Una última opción, siempre disponible, consiste en las visitas que utilizan medios auditivos (audioguías) o son autodirigidas por el adulto que acompaña a los niños en sus visitas. Esta opción puede ofrecer información fragmentada, incompleta y no siempre pertinente para el grupo etario y no se ofrece como una actividad estructurada en los museos visitados.

\section{DISEÑANDO DESDE EL MUSEO DEL CARIBE}

Con el objetivo de diseñar un nuevo portafolio de actividades para primera infancia en el Museo del Caribe, la Corporación Parque Cultural del Caribe decidió adoptar un enfoque de innovación y apropiación social del conocimiento, entendiendo que, tal como lo plantea Colciencias (2010), la innovación comprende la efectiva incorporación social del conocimiento en la solución de problemas, o en el establecimiento de nuevas relaciones; en este sentido, la innovación a través de la apropiación no es más que la interacción entre grupos, artefactos, culturas sociales de expertos y no expertos

A partir de ese objetivo inicial se planteó, el proyecto “Diseño de actividades y recursos de primera infancia para un portafolio educativo concertado" que comprendió dos etapas de desarrollo:

1. Encuentro: Durante esta etapa se hicieron avances en la indagación sobre dos fuentes de referencia en torno a las actividades de primera infancia que incluyen: una exploración de la oferta en primera infancia ofrecida por museos relevantes de distintos campos (arte, historia, ciencia y juego) con el fin de detectar algunas tendencias por la frecuencia con la que se presentan, los recursos que utilizan y estrategias de mediación propuestas; además, una exploración alrededor de las expectativas de padres y profesiona-les de educación para la primera infancia. Este "encuentro" supuso compartir con padres, profesores, psicólogos y otros profesionales participantes, los hallazgos sobre el estado del arte en oferta educativa para primera infancia, de manera que el diálogo en torno a unas posibles actividades, se enriqueciera con este nuevo insumo.

2. Creación: Una vez se llevaron a cabo las consultas y exploraciones, se continuó hacia a una fase de diseño, en la cual se invitó a participar profesionales en diseño gráfico, diseño industrial, psicólogos y el equipo de intérpretes y profesionales del Área Educativa del Museo del Caribe. El objetivo durante esta etapa fue convocar el apoyo de otras disciplinas para diseñar material educativo. Con unos lineamientos derivados de los análisis realizados en la etapa anterior, los equipos de diseñadores tuvieron en sus manos convocatorias puntuales para diseñar material que tenía como meta satisfacer las necesidades manifestadas por padres y profesionales, a la vez que enmarcarse en la filosofía educativa del Museo del Caribe y añadir valor a las tendencias detectadas en otros museos alrededor del mundo. Con los lineamientos traducidos en diseño y prototipos funcionales se da paso a la producción de material inédito, producido a la medida del contexto.

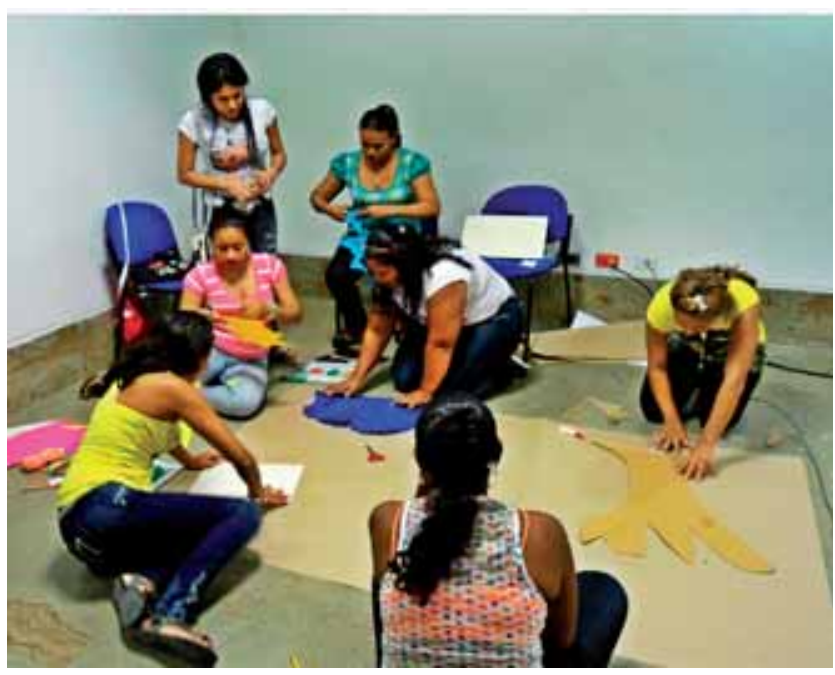

Madres y docentes de Primera infancia diseñando prototipos Laboratorio de diseño de materiales educativos (3 de marzo de 2012) FUENTE: Corporación Parque Cultural del Caribe, Archivo Fotográfico

Siguiendo la concepción apropiación del conocimiento declarada en la Estrategia Nacional de Apropiación Social de la Ciencia, la Tecnología y la innovación (Colciencias, 2010) a través del desarrollo de este proyecto, se mantuvo una conciencia alrededor de la producción de conocimiento como una construcción que no es ajena a la sociedad, sino que se desarrolla dentro de ella, a partir de sus intereses, códigos y sistemas. Por ello, la importancia de la participación de actores desde diferentes disciplinas, pero también, desde diferentes escenarios de necesidades y conocimiento, que rara vez interactúan en discusiones alrededor del diseño de actividades educativas.

Los museos mantienen, como idea, que cada exposición debe estar en consonancia con el perfil del usuario común, no de un usuario ideal o abstracto, si bien al inicio el conocimiento de sus niveles y estilos cognoscitivos sea mínimo o preliminar. Uno de los desafíos de cualquier museo de la cuarta generación es la realización de investigación sistemática permanente que permita afinar en el futuro el conocimiento específico de sus propios usuarios, para adecuar de manera idónea sus diversos elementos conforme su perfil cognoscitivo y cultural. (Orozco, 2005, p.40). En publicaciones recientes, sobre el diseño de espacios y ac- 


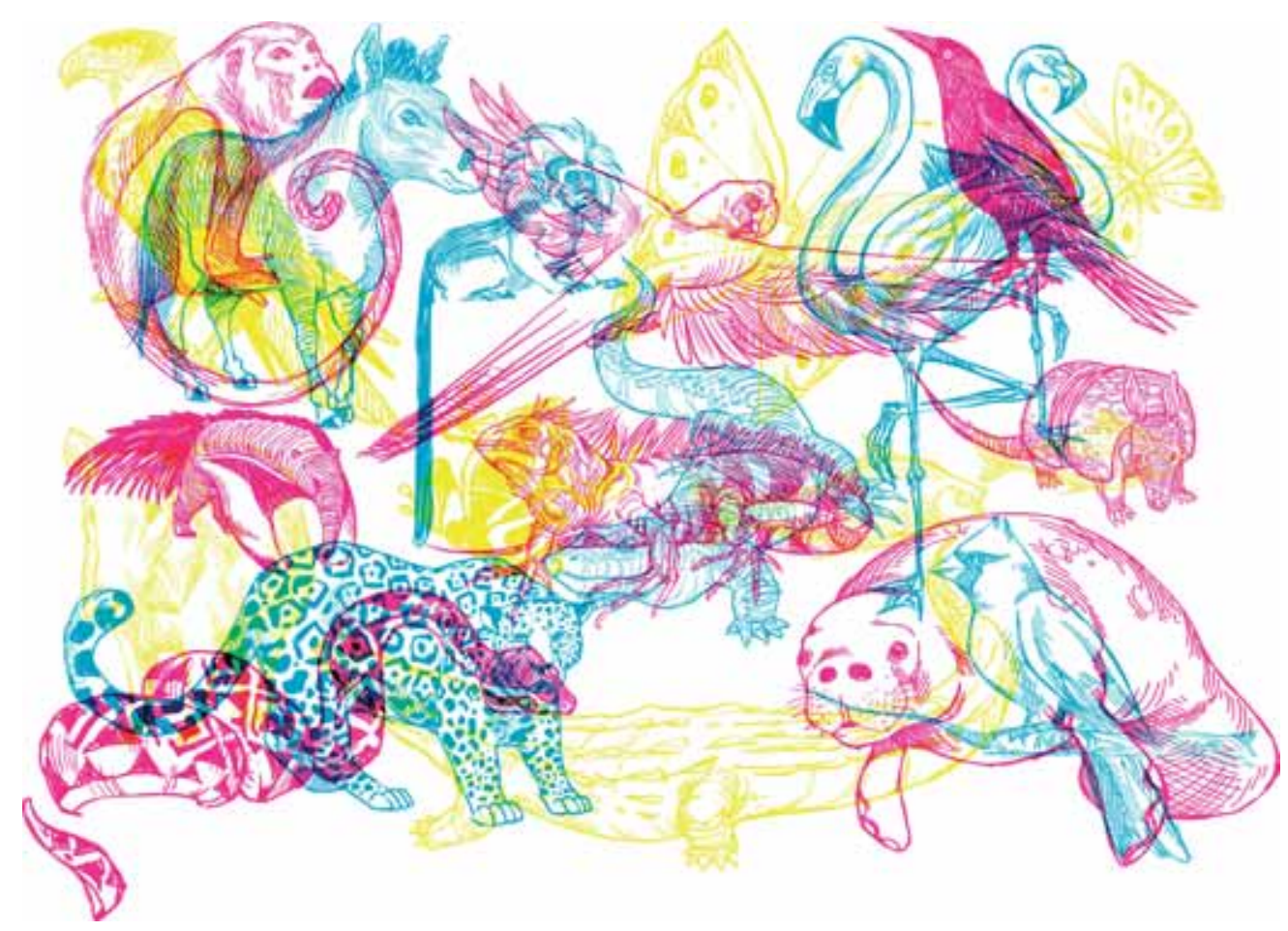

tividades en museos y centros de ciencias para niños y sus padres, como las realizadas por Vicky Cave, se ha empezado a resaltar con más frecuencia el valor de explorar ideas y conceptos [de los niños y sus familias] para que el contenido de una exhibición tienda a desplegar el potencial de los significados, lo cual puede conducir a una mayor profundidad en una exhibición. (Cave, 2010).

\section{FASE 1- ENCUENTRO: MUSEOS, MAESTROS, FAMILIAS}

Este proyecto constituyó uno de los componentes de la propuesta presentada al Ministerio de Cultura de Colombia a través de su línea de espacios concertados en 2011; aprobado por el Ministerio, y apoyado en su desarrollo durante el 2012. En la etapa de "Encuentro" se dieron cita madres de niños de primera infancia, educadores y especialistas de diversas instituciones dedicadas a sí mismo al trabajo con niños de cero a cinco años, con quienes se concertaron los primeros espacios, desarrollando lo que el equipo del Museo del Caribe ha llamado "Laboratorios de diseño de materiales educativos", cuya primera versión se desarrolló en el marco de este proyecto. En este Laboratorio, se presentaron los resultados de esa revisión de tendencias de actividades en Museos, y se construyeron con los asistentes, unas propuestas que posteriormente, el equipo de Educación del Museo del Caribe sistemazó, con el fin de obtener unos lineamientos de diseño, acorde con las expectativas de madres y maestros:
Se muestra el Mapa de Lineamientos de diseño para primera Infancia obtenido en el Laboratorio de diseño de materiales educativos por parte de la Corporación Parque Cultural del Caribe en 2012. Los Lineamientos de diseño resultantes del trabajo realizado en esta fase fueron los siguientes:

- Vivencias en torno a cuentos: aparece con frecuencia una expectativa de desarrollar sesiones con los niños, en las cuales los cuentos sean el pretexto que conduzca la actividad; cuentos narrados, juegos de rol en torno a cuentos populares, espacios que se configuren de acuerdo a las narraciones y otras actividades derivadas de las historias de tradición escrita u oral, son representativas de esta expectativa de padres y maestros.

- Lúdica: A diferencia de los maestros y padres de niños de grados escolares, los adultos vinculados a la primera infancia dan menor relevancia a los contenidos desarrollados en las actividades y privilegian por el contrario las actividades que facilitan la exploración, el disfrute, el juego y el contacto con las artes.

- Producción: El énfasis en el hacer se manifiesta reiterativamente en la preocupación de padres y maestros por que la producción, el uso de materiales como pinturas, papeles, hilos, cartón, plastilina y otros, sean puestos a disposición de los niños, y las sesiones tengan como objetivo la creación artística y concreta.

- Multisensorial: Si bien los contenidos no son una prioridad, sí lo son la activación de los sentidos y la exploración. Actividades que permitan juegos con luces, soni- 
dos, olores y texturas merecen ser destacadas para esta etapa del desarrollo.

- Espacio estructurado: Si bien padres y maestros consideran que es natural una oferta de actividades desde el Museo, también es cierto que esperan la materialización de un espacio diseñado a la medida de este público, que permita la inmersión a través de la instalación de una infraestructura específica para los niños menores de cinco años.

- Actividades cortas: Con respecto al tiempo de duración, madres, padres y maestros manifestaron en su mayoría, que las actividades para estos chicos deberían tener una duración entre 15 y 30 minutos, lo suficiente para desarollar la actividad y mantenerlos atentos e implicados.

\section{FASE 2 - CREACIÓN: DISEÑADORES, INVESTIGADORES, EDUCADORES}

Definidos los anteriores lineamientos, el siguiente paso lo constituyó la fase de creación: cuatro propuestas fueron estructuradas, tratando de responder a las expectativas manifestadas durante el Laboratorio:

1. Pinta tu ruta: Un espacio efímero que se convierte en campo de exploración y creación. Esta actividad requiere la creación de un laberinto de dimensiones variables que se puede ensamblar dentro y fuera del Museo, permite que los chicos entren y salgan plasmando en sus paredes los colores del Caribe y las figuras de su imaginación.

2. Enigma Animal: Los animales de la región Caribe se convierten en un pretexto para iniciar la exploración visual de un mural lleno de colores. Esta experiencia se desarrolla en un espacio particular, que permite controlar la luz que se refleja sobre el mural, y con ella los animales que nos son visibles. El objetivo de esta experiencia remite a la exploración visual de los más pequeños, apoyados por sus padres, mientras aparecen y desaparecen los animales en un juego de luces y colores.

3. Los secretos de Melquíades: Directamente desde los confines de Macondo los niños reciben una visita de Melquíades, quien carga en su mochila objetos curiosos y mágicos, prestos a ser vistos, tocados, olidos y sentidos. Una recuperación de las figuras atávicas del abuelo y el viajero, que se unen para compartir con los más pequeños y sus padres historias de aventura en el Caribe cercano.

4. Embárcate: Agua, luz y color. Tres elementos fundamentales cimentan una experiencia en la que la tradición de cuentos y leyendas es acercada a los más pequeños desde un juego de linternas y sombra mágicas que componen el telón y el teatro de las historias de río, mar y agua. Con unas linternas diseñadas especialmente para la actividad por los miembros del equipo de investigación Ellipsis, de la Universidad Autónoma del Caribe, se trabajó en el diseño de estos recursos.

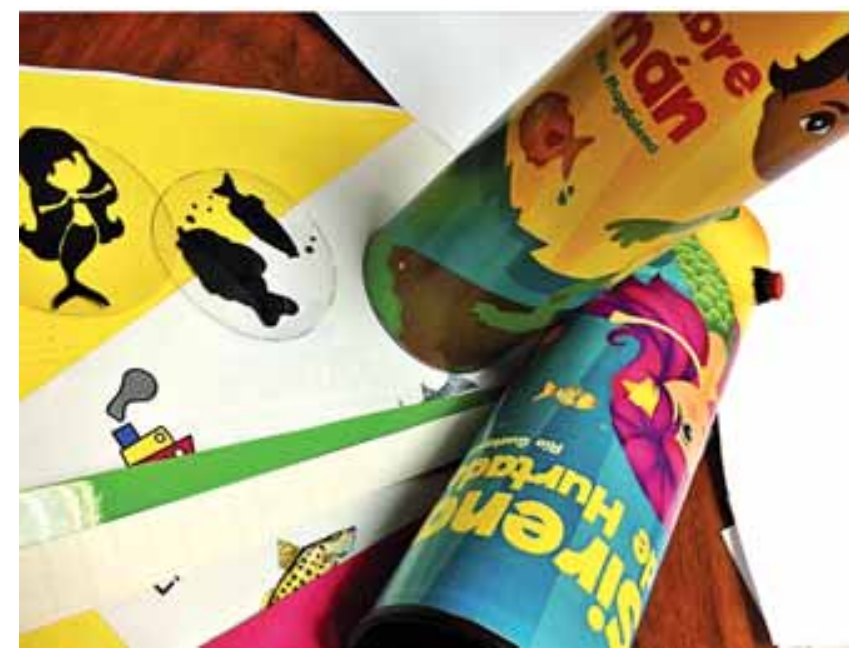

Los museos tienen la capacidad de crear experiencias memorables, significativas y altamente contextualizadas, es en estas vivencias donde se detonan en los públicos, procesos de aprendizaje que permiten que el patrimonio sea resignificado, valorado y apropiado por los usuarios. El éxito de la difusión, comunicación y exhibición de los bienes tangibles e intangibles depende directamente de la comprensión de los intereses, estilos y expectativas de las audiencias.

El Museo del Caribe, como institución de educación informal, desde su dimensión de constructo social se concibe como un centro en que se fomentan procesos de enseñanzaaprendizaje, procurando exponer al público información para formar-informar, conservando el patrimonio cultural del Caribe Colombiano de modo atractivo, siendo así un aula-laboratorio de aprendizaje para todos, mediados por diferentes espacios multimedia y siendo un lugar de deleite estético e inspiración creativa que va a la vanguardia del Siglo XXI.

Entre los propósitos actuales del Museo del Caribe, se encuentra el de convertirse en un espacio en el que los visitantes no sólo construyan su propio conocimiento sino que de manera proactiva construyan lo que el museo es en sí. Para ello, se están implementando diferentes laboratorios educativos que den luces de las expectativas, conocimientos y motivaciones que los visitantes poseen con respecto de lo que el Museo del Caribe es. En esta oportunidad, el primer laboratorio de diseño realizado apuntó al desarrollo de actividades para niños menores de edad, motivado en la creación del primer portafolio educativo para la primera infancia, respondiendo a que éste 
es un público privilegiado en todas las organizaciones a nivel mundial. Así como lo dice la OEA (2007), se viene asumiendo que la primera infancia es una fase decisiva en el ciclo de la vida del ser humano y su atención integral permite enfrentar los retos de la pobreza, la inequidad y la exclusión social, es indudable que en estos primeros años se establecen las bases para el desarrollo del pensamiento y lenguaje que afecta el desenvolvimiento en la vida de cualquier ser humano.

Existe una creciente conciencia alrededor de los espacios significativos de aprendizaje, que deben tener un punto de partida, ya sean las personas, los educandos, que en el caso de los museos interactivos contemporáneos son niños y jóvenes, principalmente. $Y$ hay que hacer hincapié en que no es ni el contenido ni la institución, él o los puntos de partida, como se acostumbra de manera tradicional en otras situaciones y esfuerzos de educación. En la actualidad, se privilegian los espacios de pensamiento donde los niños desarrollen sus competencias en un ambiente que fomente la democracia, la autonomía, la singularidad, la apertura y la trascendencia de quienes están en formación.

En este sentido, el laboratorio de diseño posibilitó el desarrollo de un trabajo interdisciplinario entre padres de familia, educadores y psicólogos para diseñar actividades educativas en el Museo del Caribe, con una nueva arquitectura educativa donde prima los intereses y las necesidades de los niños que estarán inmersos en estas experiencias significativas de aprendizaje, al igual que la de sus padres y maestros, quienes acompañan activamente el proceso. Como resultado, se obtuvieron unos lineamientos de diseño, que permitieron al equipo de educación del museo y a equipos de investigación de diseñadores gráficos e industriales concretar cuatro actividades que son: pinta tu ruta, enigma animal, secretos de Melquíades y Embárcate.

Se espera que este primer acercamiento al desarrollo de una metodología interdisciplinaria, con énfasis en la participación de diferentes actores implicados, se desarrolle como un estilo de trabajo incluyente que vincule experiencias y genere propuestas pertinentes para el público implicado.

\section{REFERENCIAS BIBLIOGRÁFICAS}

Alderoqui, S (2008). Juventud y museos: el efecto mariposa. Museos, educación y juventud. Memorias del V Encuentro Regional de América Latina y el Caribe sobre Educación y Acción Cultural en Museos CECA - ICOM. Ministerio de Cultura. Bogotá. Consultado el 15 de septiembre de 2012 desde: http://www.museoscolombianos.gov.co/inbox/files/docs/memoriaeducacionjuventud.pdf Ancheta, A (2008). Hacia una nueva concepción de la educación de la primera infancia como derecho: avances y desafíos globales. Revista Iberoamericana de Educación. España, Universidad de Valencia.

Biggs, J. (2003). Teaching for Quality Learning at University. Buckingham, The Society for Research into Higher Education \& Open University Press.

Cave, V. (2010). The New Museum Community: Audiences, Challenges,
Benefits. Capítulo: Planning for Young Children and Families in Museums. Londres

Colciencias (2010). Estrategia Nacional de Apropiación Social de la Ciencia, la Tecnología y la innovación. Bogotá.

Dufresne-Tassé, C (2008). Análisis de 50 años de educación museal. Canadá

Jaramillo, M (2008). Museos, educación y juventud. Memorias del $\checkmark$ Encuentro Regional de América Latina y el Caribe sobre Educación y Acción Cultural en Museos CECA - ICOM. Ministerio de cultura. Bogotá. Consultado el 15 de septiembre de 2012 desde: http://www.museoscolombianos.gov.co/inbox/files/docs/memoriaeducacionjuventud.pdf

Ministerio de Educación Nacional (2009). Desarrollo infantil y competencias en la primera infancia. Bogotá, Colombia.

Ojeda, C (2008). La visita al museo como experiencia. Metodología para la medición del grado de satisfacción del visitante. Revista de los museos de Andalucía. España

Orozco, G (2005). Los museos interactivos como mediadores pedagógicos. Revista Sinectica 26. Guadalajara, México

Prince, M. (2004). Does Active Learning Work? A Review of the Research. Journal of Engineering Education. 